\title{
The Impact of Investment on Price Competition in Unlicensed Spectrum
}

\author{
Hang Zhou \\ Mobile and Communications Group \\ Intel Corp \\ hang.zhou@intel.com
}

\author{
Randall A. Berry, Michael L. Honig \\ EECS Department \\ Northwestern University \\ \{rberry, mh\}@eecs.northwestern.edu
}

\author{
Rakesh Vohra \\ University of Pennsylvania \\ rvohra@seas.upenn.edu
}

\begin{abstract}
Opening "prime" spectrum to unlicensed usage can lower the costs for offering wide area wireless services but may also lead to greater congestion due to multiple providers operating in the same band. To mitigate congestion, service providers may invest in more infrastructure or better technology. The costs of such investments must be weighed against the potential gains in revenue, which in turn will depend on the investments of other competing providers in the shared spectrum. This paper studies such trade-offs for multiple competing providers in a common unlicensed band. We extend models from earlier work on price competition with congestible resources to include the investment decisions of service providers using a common band of spectrum. Several models are considered to capture different ways investment might impact the congestion due to both a provider's own customers and those of other providers. For each model, we study a game in which providers decide on both the level of investment and the price of their service. Interestingly, in most cases, the equilibrium of the resulting game is shown to be that only a single provider invests and charges monopoly prices. On the other hand, multiple providers may enter the market when the dominant effect of investment is to reduce congestion due to the customers of other providers.
\end{abstract}

\section{INTRODUCTION}

Motivated in part by the success of $\mathrm{WiFi}$, there has been interest in making "prime" spectrum available for unlicensed use, such as the recently opened up TV white spaces [1]. Due to its better propagation characteristics, such spectrum can enable a service provider (SP) to offer wide area coverage similar to that provided by cellular operators in licensed bands. Moreover, since it is open for any SP to freely use, unlicensed spectrum can substantially lower the entrance cost for providing such services. However, better propagation also increases the potential that customers of an SP in such open spectrum will experience congestion due not only to other customers of the same SP but also due to customers of other SPs operating in the same band. To reduce such congestion, SPs can invest in better technology and/or more infrastructure. The expense of such investments must be balanced by the revenue an SP would gain in drawing customers from other SPs, who in turn may also be making investment decisions. Studying such interactions is the goal of this paper.

We build on a framework from [2] for competition among SPs with unlicensed spectrum, which in turn was based

This research was supported in part by NSF under grants CNS-0905407 and CNS-1147786. on models for price competition with congestible resources developed in the operations and economics literature (e.g., [3][5]). In this framework, SPs compete for a common pool of customers by announcing service prices. Customers in turn select an SP based on a delivered price, which consists of the announced price plus a congestion cost. A key assumption in [2] is that all customers in an unlicensed band experience the same congestion cost, which depends on the total traffic in that band across all SPs. This is motivated by the likelihood that customers sharing the unlicensed band within a given region will interfere with each other even if they are associated with different SPs. It was then shown that the resulting equilibrium profit in the unlicensed spectrum is zero. ${ }^{1}$ Since providers make no profit, it is not clear that they would have an incentive to invest to offer service in the first place. Here we explicitly model this investment choice. Moreover, by varying their investment, we allow providers to differentiate their service instead of offering the same service as in [2].

There are a variety of ways an SP can invest such as adding new infrastructure or upgrading technology in existing infrastructure. Furthermore, these investments may have different effects on the congestion seen by an SP's own traffic and the traffic of other SPs. For example, if SPs invest in base station sectorization in a cellular-type system, then investment would reduce the (uplink) congestion caused by both its own traffic and that of the other SPs (since a smaller fraction of the overall traffic would be seen in each sector). As another example, if the investment corresponds to adding more access points with a smaller footprint in a given area, then the investment of an SP will not only decrease the congestion contributed by its own traffic, but also the congestion it causes to the other SPs (since its users would be transmitting at lower power). In other instances, the investment of an SP could have a larger effect in reducing the congestion caused by its own traffic, or conversely, the effect due to other SPs' traffic, e.g., if the investment is more sophisticated user scheduling or interference mitigation, respectively. Here, we consider four stylized models that highlight some of these different effects. ${ }^{2}$

We focus on investment and price competition among

\footnotetext{
${ }^{1}$ If sunk cost is considered, then the equilibrium price will fall to be equal to the sunk cost and SPs' profit is still zero.

${ }^{2}$ Preliminary work for one of these models of investment was presented in
} [6]. 
SPs who offer service using unlicensed spectrum. For each investment model, we consider a corresponding investment and pricing game. We characterize the equilibria of these games and examine how different models influence the incentives of SPs to invest in unlicensed spectrum. Interestingly, for three of these models, if a pure strategy equilibrium exists, it is for only one provider to enter the market and to act like a monopolist. In such cases, though open spectrum lowers barriers to entry, it does not result in increased competition. This can be attributed to the congestable nature of this resource, which provides a different type of barrier to entering the market. In one model, we do find an equilibrium where multiple providers may enter the market. This corresponds to a model where the dominant effect of investment is to reduce congestion due to the customers of other SPs, making the shared resource look more like an exclusive resource for each provider.

In terms of related work, price and investment (or similarly "capacity") competition between firms has been extensively studied including the Edgeworth-Bertrand game [7] and models that build on that [8]-[10]. Two main types of models have been considered: ones in which firms decide on quantities and prices sequentially and ones in which firms simultaneously set quantities and prices. Here, we consider a sequential approach; this is more reasonable for cases where investment happens on a slower time-scale than pricing decisions, which seems appropriate for wireless services. Also, in the simultaneous case, pure strategy equilibria often do not exist, complicating the analysis. A key difference of this line of work from our model is that congestion externalities are not explicitly considered. Closer to our model is work such as [3], [5], [10]-[13], which incorporate investment into models of price competition with congestible resources. The main difference from our work is that in all of these papers each firm has access to an exclusive resource. Here, the service from each SP is provided through a non-exclusive resource since all SPs can freely use the same unlicensed spectrum. Thus, the congestion suffered by customers of each SP also depends on other SPs' customers. We shall see this assumption can fundamentally change the structure of the competitive outcome.

The rest of the paper is organized as follows. Our models of investment and price competition are described in Section II. In Section III, we characterize the investment and pricing decisions of a monopolist. The subgame-perfect equilibria for each model of investment are characterized and compared in Section IV. Many detailed proofs are omitted due to lack of space. We conclude in Section V.

\section{MOdEL}

As in [2], we consider a set of SPs who compete for a common pool of customers in part by setting prices for their services. The customers respond according to both the service prices and congestion costs of the SPs. All SPs offer service in a single common unlicensed band so that the congestion experienced by a customer of each SP depends not only on the SP's own customer mass, but also on the customer masses of other SPs. Different from [2], we allow SPs to determine an investment level that influences the congestion costs. We more precisely define this model next.

\section{A. Service Providers}

Let $\mathcal{N}=\{1, \ldots N\}$ denote the set of SPs. Each SP $i$ chooses an investment level $I_{i}$ and a corresponding price $p_{i}$ for its service. The congestion cost experienced by customers of each SP $i$ is given by $\tilde{g}_{i}(\mathbf{x}, \mathbf{I})$, where $\mathbf{x}=\left(x_{1}, x_{2}, \ldots, x_{N}\right)$ and $\mathbf{I}=\left(I_{1}, I_{2}, \ldots, I_{N}\right)$ are the vector of customer masses and investment levels of all the SPs, respectively.

An increase in investment by an SP reduces the congestion its customers experience, and hence allows it to raise its service price (due to better quality of service) or admit more customers. On the other hand, such investments incur a cost. Thus the trade-off for the SPs is between the increased revenue and the cost of investment. Here we consider a setting where all SPs are investing in similar technologies, meaning that each SP's congestion function depends on investment in the same way. This dependency is given by one of four models described next. In each case the congestion function is defined in terms of an underlying function $g: \mathbb{R} \mapsto \mathbb{R}$, which maps the "effective load" an SP's customers see to a congestion value. Different investment models simply change how this effective load is calculated.

Model (I): An SP's investment reduces the effective load caused by the traffic of all SPs in the unlicensed band, i.e.,

$$
\tilde{g}_{i}(\mathbf{x}, \mathbf{I})=g\left(\frac{\sum_{i \in \mathcal{N}} x_{i}}{I_{i}}\right) .
$$

Model (II): Each SP's investment reduces the load due to its own traffic for every SP, i.e.,

$$
\tilde{g}_{i}(\mathbf{x}, \mathbf{I})=g\left(\sum_{i \in \mathcal{N}} \frac{x_{i}}{I_{i}}\right) .
$$

Note that in this model, every SP experiences the same congestion cost and an SP's congestion depends on the investment of every other SP.

Model (III): An SP's investment only reduces the load due to its own traffic and has no effect on other SPs' traffic, i.e.,

$$
\tilde{g}_{i}(\mathbf{x}, \mathbf{I})=g\left(\frac{x_{i}}{I_{i}}+\sum_{j \neq i} x_{j}\right) .
$$

Model (IV): An SP's investment reduces the load due to other SPs' traffic so that

$$
\tilde{g}_{i}(\mathbf{x}, \mathbf{I})=g\left(x_{i}+\frac{\sum_{j \neq i} x_{j}}{I_{i}}\right) .
$$

In each model, the investment of each SP is chosen from the set $\{0\} \cup[1, \infty)$. Namely, if an SP invests a positive amount, it must invest at least 1 , which can be viewed as the minimal investment required to offer service. If SP $i$ sets investment $I_{i} \geq 1$, we refer to it as being active. On the other hand, SP 
$i$ is inactive if it chooses not to invest, i.e., $I_{i}=0$. If an SP is inactive, then its profit is zero. ${ }^{3}$

\section{B. Customers}

We assume a unit mass of infinitesimal customers all of whom choose an SP based on the delivered price, which is the sum of the price announced by an SP and the SP's congestion cost, e.g., the delivered price of SP $i$ is $p_{i}+\tilde{g}_{i}(\mathbf{x}, \mathbf{I})$.

The traffic demand for services is governed by a downward sloping demand function $D(p)$ with the inverse function $P(q)$, i.e., $P(q)$ indicates the delivered price at which a mass of $q$ customers is willing to be served. Customers always choose service from the SP with the lowest delivered price. When facing the same delivered price from multiple SPs, customers are assumed to randomly choose one of the SPs. Thus all SPs who are serving customers must have the same delivered price and this must be no greater than the delivered price of any other SP. Formally, given investment and price vectors of every $\mathbf{S P},(\mathbf{I}, \mathbf{p})$, the induced non-negative demand vector $\mathbf{x}$ is required to be a Wardrop equilibrium [14], i.e., to satisfy

$$
\begin{array}{ll}
p_{i}+\tilde{g}_{i}(\mathbf{x}, \mathbf{I})=P(X) & \text { if } x_{i}>0 \\
p_{i}+\tilde{g}_{i}(\mathbf{x}, \mathbf{I}) \geq P(X) & \text { if } x_{i}=0
\end{array}
$$

where $X=\sum_{i \in \mathcal{N}} x_{i}$ is the total customer mass being served.

In general a Wardrop equilibrium may not exist. To ensure that it does and is unique for a given investment and price vector, we make the following assumptions: ${ }^{4}$

Assumption 1: $g(x)$ and $x g(x)$ are strictly increasing and convex; $P(q)$ is concave decreasing and $q P(q)$ is strictly concave. In addition, $g(0)=0$ and there exists $Q>0$ such that $P(Q)=0$.

\section{Two-stage Investment-Pricing Game}

We consider an investment-pricing game in which each SP $i$ seeks to maximize its profit given by

$$
\Pi_{i}\left(I_{i}, \mathbf{I}_{-i}, p_{i}, \mathbf{p}_{-i}, \mathbf{x}\right)=p_{i} x_{i}-c I_{i}
$$

where $\mathbf{I}_{-i}$ and $\mathbf{p}_{-i}$ denote the investment levels and prices of the other SPs, respectively, and $\mathrm{x}$ is the resulting demand vector that satisfies (1). The first term in (2) represents the revenue collected from $i$ 's customers, and the second term represents its investment cost, which is assumed to be proportional to $I_{i}$ with constant marginal investment cost $c .{ }^{5}$

Each provider determines its investment and pricing decisions sequentially, i.e., this is a two-stage game in which the SPs first simultaneously set investment levels $\left\{I_{i}\right\}$. Having observed the investment decisions of all SPs in the first stage, the SPs then announce their service prices $\left\{p_{i}\right\}$ simultaneously

\footnotetext{
${ }^{3}$ Note that in all these models the congestion costs are not well-defined when $x_{i}=0$ and $I_{i}=0$ for each $i$. Thus to formally define an equilibrium in such cases, we view $\tilde{g}_{i}(\cdot)$ as being so large that no customers would want to use the service of SP $i$.

${ }^{4}$ With these assumptions, the existence of a unique Wardrop equilibrium can be shown by using an argument as in [15].

${ }^{5}$ Here we assume that all providers have the same marginal investment costs; however, many of our results can be generalized to asymmetric investment costs at the expense of more cumbersome notation.
}

in the second stage. Since the investment set in the first stage is observed by all SPs, every investment vector $\mathbf{I}=\left(I_{1}, \ldots, I_{N}\right)$ then defines a proper subgame, which we refer to as a pricing subgame. An equilibrium for this subgame is defined next.

Definition 1: The price and demand vector $(\mathbf{p}(\mathbf{I}), \mathbf{x}(\mathbf{I}))$ is a pure strategy price equilibrium in the pricing subgame for a fixed investment vector $\mathbf{I}$ if $\mathbf{x}(\mathbf{I})$ satisfies (1) and no SP can increase its profit by unilaterally changing prices.

SPs can set investment levels in the first stage expecting to see such a price equilibrium in the second stage. This allows us to define a subgame perfect equilibrium (SPE) [16] for the overall game, which we refer to as the investment-price equilibrium.

Definition 2: A tuple $\left(\mathbf{I}^{\mathbf{e}}, \mathbf{p}\left(\mathbf{I}^{\mathbf{e}}\right), \mathbf{x}\left(\mathbf{I}^{\mathbf{e}}\right)\right)$ is an investmentprice equilibrium if $\left(\mathbf{p}\left(\mathbf{I}^{\mathbf{e}}\right), \mathbf{x}\left(\mathbf{I}^{\mathbf{e}}\right)\right)$ forms a price equilibrium in the pricing subgame given $\mathbf{I}^{\mathbf{e}}$, and no SP can increase its profit by unilaterally changing its investment.

\section{MONOPOLY SCENARIO}

In this section we characterize the investment and pricing decisions made when there is only a single monopoly SP present in the unlicensed band. ${ }^{6}$. The monopoly SP will jointly optimize its profit, $\pi=p x-c I$, over investment $I \in\{0\} \cup[1, \infty$ ) and price $p \geq 0$ (or equivalently, customer demand $x \geq 0))^{7}$ Furthermore, if there is only a single SP, it can be seen that the congestion cost functions of Models I, II and III all become the same with $\tilde{g}(\mathbf{x}, \mathbf{I})=g\left(\frac{x}{I}\right)$. For Model IV, if there is no congestion caused by other SPs, then the monopoly SP does not need to make any investment greater than 1 . Thus we focus on the monopoly behavior corresponding to Models I-III.

The monopoly's profit maximization can be written as the following problem.

$$
\begin{aligned}
\max _{I, p, x} & \Pi=p x-c I \\
\text { s.t. } & p+g\left(\frac{x}{I}\right)=P(x), \\
& x \geq 0, \quad p \geq 0, \quad I \in\{0\} \cup[1, \infty)
\end{aligned}
$$

which can be rewritten as

$$
\begin{array}{cl}
\max _{I, x} & \Pi=x P(x)-x\left(g\left(\frac{x}{I}\right)+c \frac{I}{x}\right) \\
\text { s.t. } & x \geq 0, \quad I \in\{0\} \cup[1, \infty) .
\end{array}
$$

To interpret this, note that $P(x)$ is the delivered price that $x$ customers would be willing to pay. If there was no congestion or investment, the monopolist could charge each customer $P(x)$ and have a total profit of $x P(x)$. The term $g\left(\frac{x}{I}\right)+c \frac{I}{x}$ represents the monopolist's per customer cost due to congestion and investment.

We make the following assumption so that a monopoly will always make positive profit, and hence want to enter the

\footnotetext{
${ }^{6}$ The unlicensed band essentially becomes the monopoly SP's proprietary band in this scenario.

${ }^{7}$ Since there is only one SP, whether these optimization are done sequentially or simultaneously does not matter.
} 
market. This requires that the monopoly has positive profit with investment equal to 1 . From (3), this gives the following condition.

Assumption 2: $\max _{x \geq 0} x(P(x)-g(x))>c$.

Because of Assumption 2, we only need to consider the case where the monopoly invests $I \geq 1$.

Define $\Delta(t)=g(t)+c \frac{1}{t}$ such that the second term in the objective of (3) can be expressed as $x \Delta(x / I)$. To solve (3), one can first minimize $\Delta(t)$ over $t \geq 0$, where $t=x / I$. Let the solution to this minimization be $\xi$; this must be an interior point that satisfies the first order condition $g^{\prime}(\xi) \xi^{2}=c$. Let the corresponding optimal objective value be $\Delta_{c}^{*}$, i.e., this is the minimum total cost per customer the monopolist can obtain. The monopoly investment and price are then given by the following lemma.

Lemma 1: Under Assumptions 1 and 2, the monopoly investment $I^{M}$ and customer mass $x^{M}$ are given by

$$
\left(I^{M}, x^{M}\right)= \begin{cases}(1, \chi), & \text { if }\left.\frac{\mathrm{d}}{\mathrm{d} x} x P(x)\right|_{x=\xi} \leq \Delta_{c}^{*}, \\ \left(I_{0}, x_{0}\right), & \text { if }\left.\frac{\mathrm{d}}{\mathrm{d} x} x P(x)\right|_{x=\xi}>\Delta_{c}^{*},\end{cases}
$$

where $\chi$ satisfies $\left.\frac{\mathrm{d}}{\mathrm{d} x}(x P(x)-x g(x))\right|_{x=\chi}=0 ; x_{0}$ satisfies $\left.\frac{\mathrm{d}}{\mathrm{d} x} x P(x)\right|_{x=x_{0}}=\Delta_{c}^{*}$ and $I_{0}=x_{0} / \xi$. The corresponding monopoly price is given by (1).

Here $\frac{\mathrm{d}}{\mathrm{d} x} x P(x)$ represents the marginal gain from serving one more customer. The Lemma shows that one will invest at a level larger than 1 only if this marginal gain exceeds $\Delta_{c}^{*}$. The proof of this follows from simply interpreting the first order optimality conditions of problem (3) and is omitted.

\section{Oligopoly Scenario}

Next, we turn to the scenario where $N \geq 2$ SPs compete for customers. For each model of investment, we first characterize the investment price equilibria. Before preceding, we first make the following assumption on the customer response to SPs' prices, in addition to (1).

Assumption 3: Any SP $j$ with $p_{j}=0$ will receive $x_{j}=0$ whenever its delivered price is the same as that of some other SP $i$ with $p_{i}>0$.

The purpose of Assumption 3 is to guarantee the existence of a pure strategy price equilibrium. The conditions for this assumption will never occur on the equilibrium path, since an active SP with a price of zero will make zero profit and thus should not invest in the first place. However, it is needed to guarantee the existence of pure strategy equilibrium for pricing sub-games off the equilibrium path.

\section{A. Equilibria of Model I}

For model I, it can be shown that if more than one SP had a positive price and customer mass, one of the SPs can always increase its profit by lowering its price and attracting customers away from the other SPs. If all providers invest the same amount, then as in [2] the resulting "price war" will lead to zero prices and negative profits, showing that this cannot be an equilibrium. However, if one SP invests a larger amount than any other SPs, it will have a lower congestion cost and thus can "win" this price war while still sustaining a positive price. This shows that the only potential investment -price equilibria are ones in which there is only one active SP. This is summarized in the following Theorem, which also gives a sufficient condition for such an equilibrium to exist.

Theorem 1: For investment model 1, in any investmentprice equilibrium there can be at most one active SP and this SP must be using the monopoly price $x^{M}$ and investment level $I^{M}$. Moreover, let $\underline{x}$ be such that $P(\underline{x})=g\left(\frac{x}{I^{M}}\right)$. If $P(\underline{x}) \leq \Delta_{c}^{*}$, such an equilibrium will exist.

Here $\underline{x}$ can be interpreted as the customer mass a monopolist would serve with zero announced price and $\Delta_{c}^{*}$ is as defined in Lemma 1. To show such equilibrium exists, one needs to show that given the monopoly's investment and price, other SPs do not have an incentive to enter the market, i.e., entering the market will result in negative profit. The condition involving $\underline{x}$ provide a sufficient condition for this to be true.

\section{B. Equilibria of Model II}

For model II, every active SP has the same congestion cost, i.e., investing more does not give an SP any advantage in terms of its delivered price. Hence if multiple SPs invest, this is similar to the model without investment in [2], where it was shown that the price for unlicensed spectrum was zero. Indeed by a similar argument as in Theorem 1 in [2] we have the following lemma:

Lemma 2: For investment model II, given an investment vector I with more than one non-zero component, there exists a unique price equilibrium $(\mathbf{p}(\mathbf{I}), \mathbf{x}(\mathbf{I}))$ and it satisfies $p_{i}=0$ for every active SP $i$.

Based on Lemma 2, we again see that there can be at most one active SP in any investment-price equilibrium. Otherwise, every active SP would receive negative profit, and hence would be better off by investing zero. Furthermore, in this case, such an equilibrium always exists since any SP would also receive a negative profit if it entered the market to compete with a monopolist. We summarize this in the following theorem.

Theorem 2: For investment model II, a pure strategy investment-price equilibrium always exists and any such equilibrium must correspond to a single active (monopoly) SP.

\section{Equilibria of Model III}

In the previous models, given the investments, the congestion seen at each SP can be ordered independent of the traffic (in the case of model 2, the ordering is trivial since the congestion is the same). For model III, this is not the case since each SP's investment only effects the load due to its own traffic on its own network.

To begin, we examine the price equilibrium with two SPs. The next lemma shows that if one SP invests strictly less than the other, then it can not have a non-zero price and customer mass in the equilibrium.

Lemma 3: For investment model III with two SPs, suppose the investments are $I_{1}>I_{2} \geq 1$. Then the corresponding price equilibrium $\left(\mathbf{p}^{e}, \mathbf{x}^{e}\right)$ is unique and satisfies $p_{1}^{e}>0, p_{2}^{e}=0$, 
$x_{1}^{e}>0$ and $x_{2}^{e}=0 .{ }^{8}$

To understand this lemma note that for model III any SP investing more than 1 will always see less congestion if a mass of customers is assigned to it as opposed to another SP, and this gain is largest for the SP with the largest investment. In particular, suppose that in a Wardrop equilibrium the two SPs both had non-zero prices and customer masses, with at least one the SPs investing more than 1. Suppose further that this SP slightly lowers its price and as a result a small fraction of customers switched to that SP. From the above observation, this would further lower the congestion (and thus the delivered price) leading to yet more customers switching, until all customers were being served by the SP who lowered its price. By making this price decrease arbitrarily small, this must increases the SP's profit, showing that the original prices can not have been a price equilibrium. It follows that in a price equilibrium at least one SP must be serving no customers. In can further be shown that this must be SP 2 because the SP 1 can always use its investment advantage to undercut any nonzero price that SP 2 sets and attract all of the customers. On the other hand, SP 1 can always set a non-zero equilibrium price which is given by maximizing $p_{1} x_{1}$ subject to the constraints:

$$
p_{1}+g\left(\frac{x_{1}}{I_{1}}\right)=P\left(x_{1}\right) \quad \text { and } \quad g\left(\frac{x_{1}}{I_{2}}\right) \geq P\left(x_{1}\right) .
$$

For $I_{1}>I_{2}$ this maximization will have a solution $p_{1}^{e}>0$ from which SP 1 will not want to deviate. Further, the second constraint ensures that SP 2 can not announce any price to undercut SP 1 . Hence this must be a price equilibrium.

Lemma 3 assumed that the investments were not equal. With equal investments $\left(I_{1}=I_{2}\right)$, as in Model II, the price equilibria will be for both SPs to announce a price of zero. Also, though we stated Lemma 3 for only 2 SPs, the result can be directly extended to more than two users in which case it follows that only the SP with the largest investment will have strictly positive price and customer mass in the price equilibrium. Therefore, again, there can be at most one SP in an investmentprice equilibrium and such an SP must be using the monopoly investment and pricing. As with Model I, to show that such an equilibrium exists requires showing that given the monopoly investment and price, other SPs cannot obtain positive profit upon entering the market. As we have noted, in the monopolist case, Models 1 and 3 become the same and so both of these models have the same price equilibria. Therefore we can again use the sufficient condition in Theorem 1 to ensure that an equilibrium exists.

\section{Equilibrium of Model IV}

So far, the only investment-price equilibria are for a single SP to act as a monopolist. In this section, we consider the final investment model and see that different equilibrium structures may arise. Recall, in this model investment reduces the congestion caused by other SPs' traffic, for example by better interference mitigation. We focus on a simple case with

\footnotetext{
${ }^{8}$ More precisely the equilibrium values of $p_{1}^{e}, x_{1}^{e}$, and $x_{2}^{e}$ are unique, $p_{2}^{e}$ can be any value.
}

two SPs whose congestion and demand satisfy the following assumption. We conjecture that similar results can be obtained for general congestion and demand functions.

Assumption 4: $g(x)=a x$ and $P(x)=T-b x$.

In Assumption 4, the parameter $a>0$ in the congestion cost is assumed to be a decreasing function of the available bandwidth in the unlicensed spectrum. The parameter $b>0$ in the demand function characterizes the total mass of customers: smaller values of $b$ implying more customers in the market.

First, we consider price equilibria. The cases with $I_{1}=0$ or $I_{2}=0$ are trivial, and if $I_{1}=I_{2}=1$, then the pricing subgame is equivalent to the game in [2] for which it is known that the equilibrium price is zero for every SP. Thus, we focus on the case where $I_{1}>I 2 \geq 1$.

Lemma 4: Suppose Assumption 4 holds. If $I_{1}>I_{2} \geq 1$ then the price equilibrium is unique and satisfies $p_{1}^{e}>0$, $p_{2}^{e}>0, x_{1}^{e}>0$, and $x_{2}^{e}>0$.

This lemma follows from noting that if $p_{1}>0$ and $x_{2}=0$, then it must be that

$$
p_{1}+g\left(x_{1}\right) \leq g\left(\frac{x_{1}}{I_{2}}\right) ;
$$

otherwise SP 2 can find a price at which it would make a positive profit. But since $I_{2} \geq 1$, this cannot be satisfied, which shows that both SP's must be serving a positive customer mass in any price equilibrium. Hence, in any price equilibrium both SPs must have the same delivered prices. Using this and Assumption 4, one can solve for the demands in terms of the prices and then solve the profit maximization facing each SP to determine each SP's best response price to a price of the other SP. Examining these best response functions, the existence and uniqueness of a price equilibrium follows.

Lemma 4 shows that a key difference in price equilibrium between Model IV and the preceding models: here there are multiple SPs with positive prices and customer masses. This suggests that investment-price equilibria with multiple active SPs may exist. Indeed, the following result shows that a symmetric equilibria (with two active SPs who invest the same and set the same price) exists for a range of parameters.

Proposition 1: If the parameters in Assumption 4 satisfy

$$
c<\frac{a^{2} T^{2}}{32(a+b)^{3}}
$$

then there exists a unique symmetric investment-price equilibrium for Model IV.

The main technical difficulty here is that the overall strategy space is not convex due to the assumed minimum investment to be active. However, using Lemma 4 one can use standard results to show the existence and uniqueness of a symmetric equilibrium in a "modified game" where agents are always active. The condition in this proposition in then sufficient to ensure that this is also an equilibrium in the original game.

The next proposition shows that an asymmetric investmentprice equilibrium (in which only one SP is active) can also exist for a certain range of parameters. 


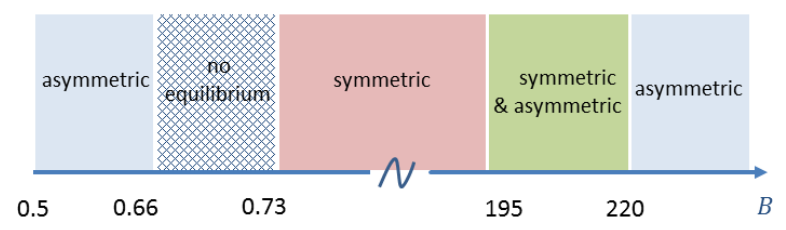

Fig. 1. The investment-price equilibria for model IV as a function of the bandwidth $B$ when $a=1 / B$ and $b=0.1$.

Proposition 2: If the parameters in Assumption 4 satisfy

$$
c>\frac{4 a T^{2}}{9(a+b)^{2}},
$$

then there is an investment-price equilibrium, in which only one SP is active with investment $I^{M}=1$. Furthermore, for small enough $a$, this is the only type of equilibrium.

This follows from simply evaluating each SP's best response when only one invests and showing that this is an equilibrium under the given condition. Comparing the conditions in Proposition 1 and 2, it can be seen that for fixed positive $c$ and $b$, an asymmetric equilibria exists for small enough or large enough $a$, while a symmetric equilibria does not exist for small enough $a$ and may not exist for large enough $a$. An example of this behavior is shown in Figure 1. This was generated from numerical results assuming that $a=\frac{1}{B}, b=0.1$, $c=0.05$ and $T=1$. Note that symmetric equilibria (with two active SPs) fail to exist not only when there is insufficient spectrum, but also when there is abundant spectrum. The reason is that both insufficient and abundant spectrum tend to reduce the incentive of an SP to invest. The issue with abundant spectrum is somewhat subtle: the problem here is that for a symmetric equilibrium, the SPs need to invest at a level greater than one; when spectrum is abundant there is no incentive for increased investment, since the nominal investment is sufficient to adequately serve all customers.

Notice that the conditions in these propositions are not mutually exclusive, i.e., symmetric and asymmetric equilibria may exist simultaneously for some parameters, which is indeed observed in our numerical results for a range of $a$. However, the following lemma rules out the possibility of the monopoly equilibria for a certain range of the parameters.

Lemma 5: If $a \geq b$ and there exists some $I \geq \frac{2 a}{a-b}$ such that the investment vector $(I, I)$ is an equilibrium, then all equilibria are symmetric.

In particular this lemma shows that for a range of parameters the monopoly equilibria does not exist. The proof follows from first noting that the only possible asymmetric equilibria are ones where one SP is a monopolist and then arguing that under the given assumptions this can not be an equilibrium.

\section{CONCLUSION}

Several models for investment and price competition in unlicensed spectrum were studied. Different investment models provided different incentives for SPs to invest, and thus led to different equilibrium market structures. In many cases, the equilibria, if one exists, corresponds to a single SP investing, i.e., a monopolist emerges. However, when the effect of investment is mainly to reduce interference from other SPs, equilibria with multiple SPs may emerge. From a policy pointof-view, if the goal of introducing unlicensed spectrum is to foster competition, then clearly a monopoly emerging is not the desirable outcome. In such a case, these results suggest that policy should also encourage investment that reduces interference from other SPs or that one should consider an alternative policy for spectrum access. On the other hand, in cases like providing broadband access in rural areas, having a monopoly emerge to provide such access may be an acceptable outcome (given that the status quo may be no access.)

The models we considered here were highly stylized. One could study several generalizations, which we leave for future work. These include more detailed modeling of congestion effects, adding uncertainty about the actions of other SPs, consider SPs who also have access to licensed spectrum, allowing providers to invest in multiple types of technologies and finally considering heterogeneous uses of such spectrum.

\section{REFERENCES}

[1] "Unlicensed operation in the TV broadcast bands ; additional spectrum for unlicensed devices below $900 \mathrm{MHz}$ and in the $3 \mathrm{GHz}$ band- second memorandum opinion and order," FCC Report and Order, September 2010.

[2] T. Nguyen, H. Zhou, R. Berry, M. Honig, and R. Vohra, "The impact of additional unlicensed spectrum on wireless services competition," in Pproceedings of Fifth IEEE International Symposium on New Frontiers in Dynamic Spectrum Access Networks 2011 (DySPAN 2011), 2011.

[3] D. Acemoglu and A. Ozdaglar, "Competition and efficiency in congested markets," Mathematics of Operations Research, vol. 32, no. 1, pp. 1-31, February 2007.

[4] - "Competition in parallel-serial networks," IEEE Journal on Selected Areas in Communications, vol. 25, no. 6, pp. 1180-1192, August 2007.

[5] R. Johari, G. Y. Weintraub, and B. V. Roy, "Investment and market structure in industries with congestion," Operations Research, vol. 58, no. 5, pp. 1303-1317, 2010.

[6] H. Zhou, R. Berry, M. Honig, and R. Vohra, "Investment and competition in unlicensed spectrum," in Proceedings of 46th Annual Conference on Information Sciences and Systems (CISS 2012), March, 2012.

[7] J. Tirole, The Theory of Industrial Organization. The MIT Press, 2001.

[8] R. Levitan and M. Shubik, "Duopoly with price and quantity as strategic variables," International Journal of Game Theory, vol. 7, pp. 1-11, 1978.

[9] D. M. Kreps and J. A. Scheinkman, "Quantity precommitment and bertrand competition yield cournot outcomes," The Bell Journal of Economics, vol. 14, no. 2, pp. pp. 326-337, 1983.

[10] D. Acemoglu, K. Bimpikis, and A. Ozdaglar, "Price and capacity competition," Games and Economic Behavior, vol. 66, no. 1, pp. pp. 1-26, 2009.

[11] M. A. Campo-Rembado and A. Sundararajan, "Competition in wireless telecommunications," Stern School of Business, New York Univerisity, Working paper, 2004.

[12] F. Xiao, H. Yang, and D. Han, "Competition and efficiency of private toll roads," Transportation Research Part B: Methodological, vol. 41, no. 3, pp. 292 - 308, 2007.

[13] B. D. Borger and K. V. Dender, "Prices, capacities and service levels in a congestible bertrand duopoly," Journal of Urban Economics, vol. 60, no. 2 , pp. $264-283,2006$.

[14] J. G. Wardrop, "Some theoretical aspects of road traffic research," Proceedings, Institute of Civil Engineers, PART II, vol. 1, pp. 325-378, 1952.

[15] T. Nguyen, H. Zhou, R. Berry, M. Honig, and R. Vohra, "The price of free spectrum to heterogeneous users," in Workshop on the Economics of Networks, Systems and Computation (NetEcon'11), 2011.

[16] A. Mas-Colell, M. D. Whinston, and J. R. Green, Microeconomics Theory. Oxford University Press, 1995. 